Supporting Information

\title{
Modeling Supramolecular Polymerization: The Role of Steric Effects and Hydrophobic Interactions
}

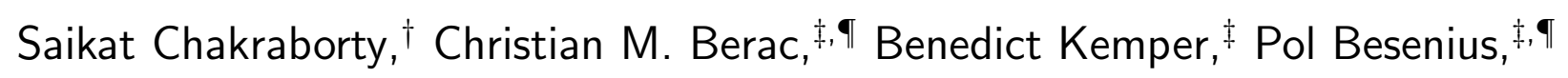
and Thomas Speck*,t,

$\dagger$ Institut für Physik, Johannes Gutenberg-Universität Mainz, Staudingerweg 7-9, 55128 Mainz, Germany

$\ddagger$ Institut für Organische Chemie, Johannes Gutenberg-Universität Mainz, Duesbergweg 10-14, 55128 Mainz, Germany

\Graduate School "Materials Science in Mainz", Johannes Gutenberg-Universität Mainz, Staudingerweg 9, 55128 Mainz, Germany

E-mail: thomas.speck@uni-mainz.de 


\section{Experimental Details}

\section{Chemical structure of monomer}

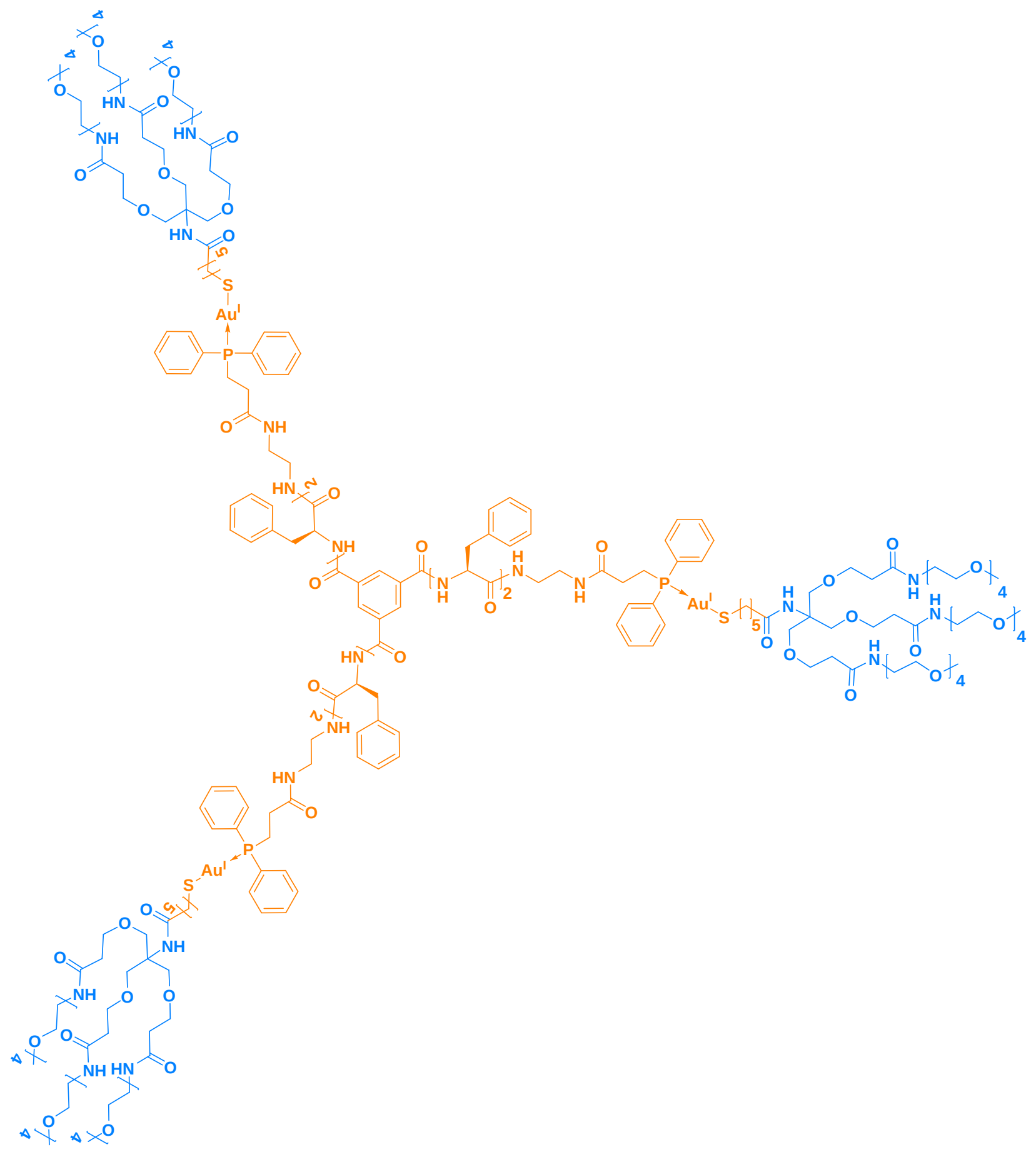

Supplemental Figure 1: Chemical structure of the amphiphilic $\mathrm{Au}^{\mathrm{I}}$-metallopeptides with a hydrophobic core and a peptide sequence (orange) and a hydrophilic oligoethylene glycol based dendritic unit (blue). 


\section{Circular dichroism (CD) spectroscopy}

All CD spectra (Supplemental Figure 2) were recorded using a monomer concentration of $10-$ $100 \mu \mathrm{M}$ in $10 \mathrm{mM}$ phosphate buffer (pH 7.4) using a quartz cell with a path length of $10 \mathrm{~mm}$, $2 \mathrm{~mm}$ or $1 \mathrm{~mm}$. CD-spectra were recorded on a J-815 CD spectrometer (JASCO) using the software Spectra Manager 2.08.04. An average of three scans was reported. All spectra
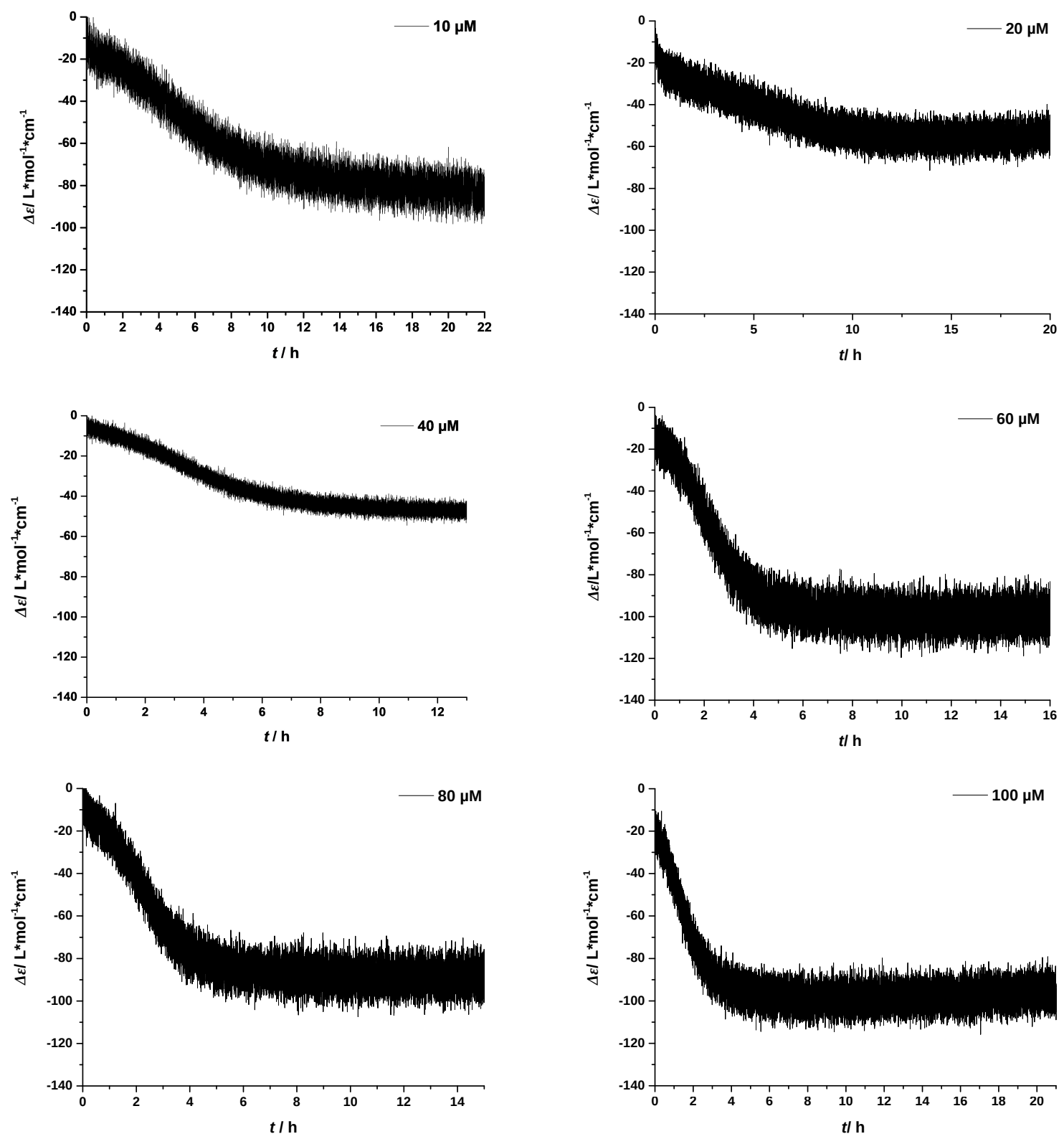

Supplemental Figure 2: Time-dependent CD spectroscopic analysis $(\lambda=219 \mathrm{~nm})$ of a freshly ice-cold prepared solution of monomers in phosphate buffer $(10 \mathrm{mM}, \mathrm{pH} 7.4)$ at constant $10^{\circ} \mathrm{C}$ and concentrations of $10-100 \mu \mathrm{M}$. 


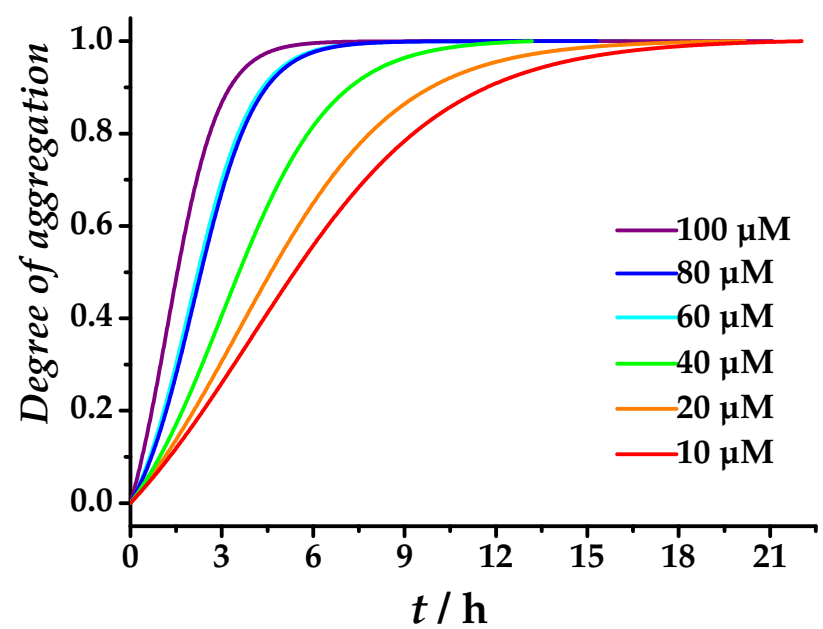

Supplemental Figure 3: Normalized and smoothed time-dependent CD spectroscopic analysis (Supplemental Figure 2).

were corrected by the subtraction of the buffer (background). All data were processed using OriginPro 9.1. For clarity of presentation of the concentration dependence of polymerization, we normalize and smoothen the curves of Supplemental Figure 2. The results are shown in Supplemental Figure 3.

\section{Transmission electron microscopy (TEM)}

TEM samples were prepared from $50 \mu \mathrm{M}$ solutions in $10 \mathrm{mM}$ TRIS buffer $(\mathrm{pH} 7.4) .5 \mu \mathrm{L}$ sample droplets were absorbed for 1 minute on freshly glow-discharged copper grids (CF300-

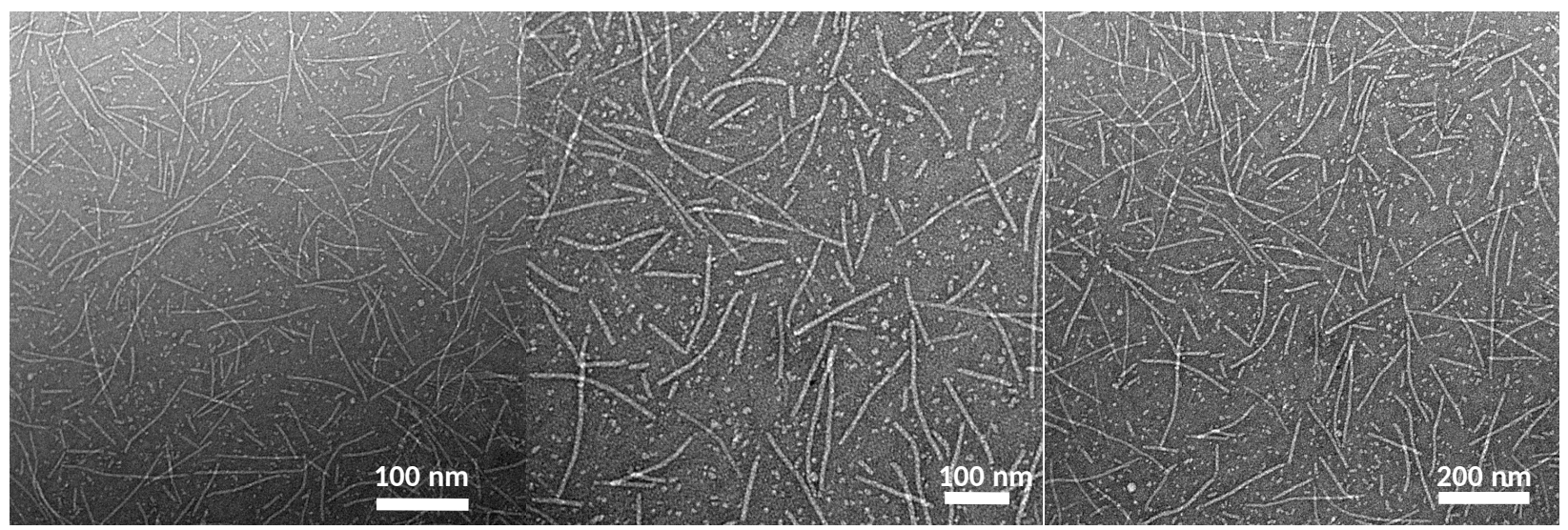

Supplemental Figure 4: Representative TEM images $(50 \mu \mathrm{M})$ in Tris buffer $(10 \mathrm{mM})$ at $20^{\circ} \mathrm{C}$. 
$\mathrm{Cu}, 300$ mesh) coated with a $3-4 \mathrm{~nm}$ carbon layer. The grids were then negatively stained using a $2 \% \mathrm{v} / \mathrm{v}$ solution of uranyl acetate for $15 \mathrm{~s}$. TEM images (Supplemental Figure 4) were accomplished on a FEI TecnaiTM T12 transmission electron microscope equipped with a BioTWIN lens and a LaB6 cathode operated at $120 \mathrm{kV}$. Digital electron micrographs were recorded with a $4 \mathrm{k} \times 4 \mathrm{k}$ CMOS camera (TVIPS).

\section{Supplementary Results from Simulations}

\section{Results for small value of interaction strength}

Results for the assembly kinetics for small values of attractive interaction strength $(\epsilon=5)$ is presented in Supplemental Figure 5. The growth profiles look similar to Figure 2 in the main text. However, at the lowest initial monomer concentrations studied, the mass fraction $M(t) / C$ does not reach unity even at very long time. This indicates that monomer dissociation does become relevant at these conditions. Clearly, the Asakura-Oosawa (AO) model $^{1}$ can not be utilized to analyze these growth profiles. Nevertheless, with growing values of $C, M(t) / C$ approaches unity and the effect of monomer dissociation does become negligible.

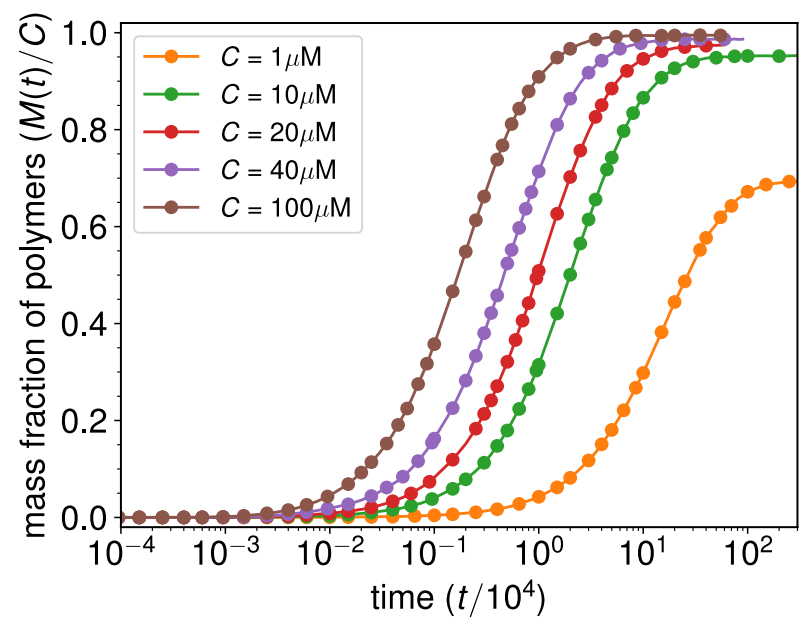

Supplemental Figure 5: Importance of monomer dissociation. Sigmoidal polymerization growth profiles for different values of initial monomer concentration $C$ with $\epsilon=5$. 

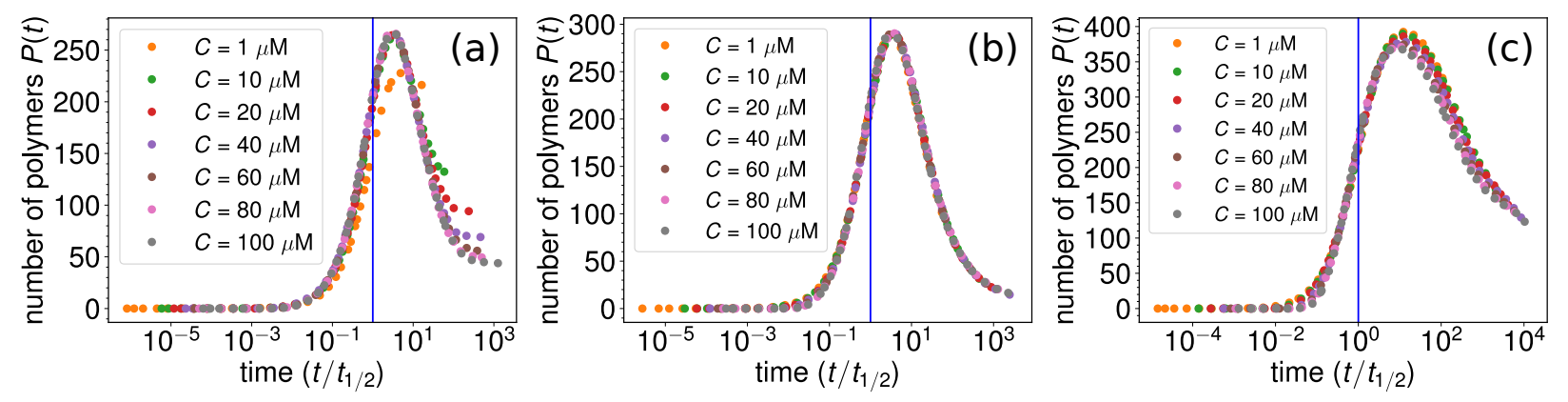

Supplemental Figure 6: Time evolution of number of polymers. Variation of polymer number $P(t)$ during self-assembly for $(\mathrm{a}) \epsilon=5,(\mathrm{~b}) \epsilon=10$, and $(\mathrm{c}) \epsilon=30$. Data for different initial monomer concentrations collapse when the time axis is scaled by $t_{1 / 2}$. The presence of monomer dissociation results in poor collapse for $\epsilon=5$. For all values of $\epsilon$, the decay starts much later than $t_{1 / 2}$ (blue vertical lines), reflecting nucleation and elongation to be major mechanisms at the initial polymerization stage so that the AO model can be applied.

\section{Time evolution of number of polymers}

Supplemental Figure 6 shows the evolution of the number of polymers $P(t)$ as a function of time for different values of the interaction strength $\epsilon$. As discussed in the main text, nucleated polymerization followed by end-to-end aggregation of polymer filaments results in the nonmonotonic behavior of the plots. In contrast to Figure $2 \mathrm{c}$ in the main text, here the time axis is scaled by $t_{1 / 2}$, collapsing data points for all initial monomer concentrations. Poor collapse for $\epsilon=5$ is again related presumably to the significant presence of monomer dissociation. Notably, for all values of $\epsilon$, the dominance of filament coalescence (decay in the plots) starts much later than $t_{1 / 2}$. Therefore, the global fitting of simulation data with AO model $^{1}$ for $t<t_{1 / 2}$ is indeed justified.

\section{Effect of finite-size}

The results presented in the main article are from simulations with number of monomers $N=1000$. The value of $N$, though apparently small, corresponds to a cubic box of linear dimension $L \cong 384 \sigma(\equiv 1.15 \mu \mathrm{m})$ for initial monomer concentration $C=10 \mu \mathrm{M}$. However, it is always instructive to check whether there is any effect of finite-size on the conclusions of the simulations. To check for that, in Supplemental Figure 7 the time evolution of the 


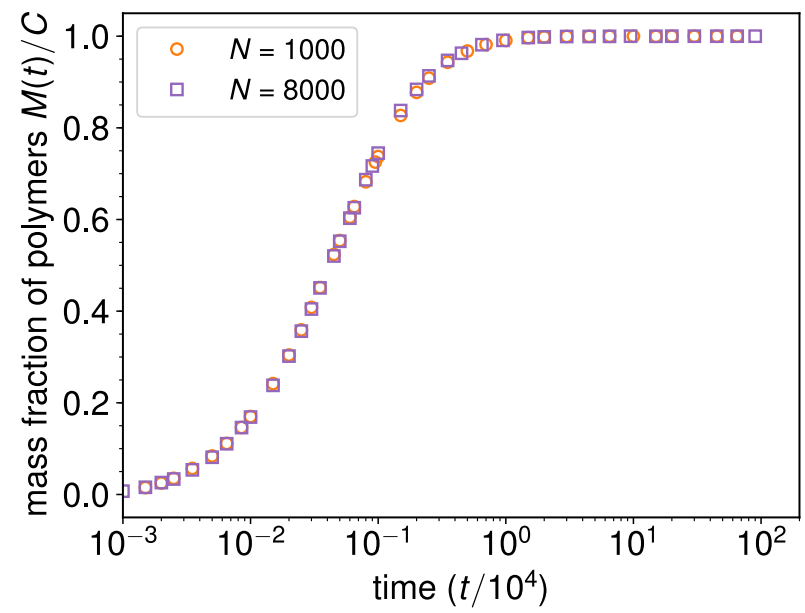

Supplemental Figure 7: Effect of finite size. Assembly kinetics at $\epsilon=10$, varying number of initial monomers keeping concentrations fixed at $80 \mu \mathrm{M}$. Almost identical growth profile indicates that the conclusions drawn in the main article is not size dependent.

mass fraction of polymers $M(t) / C$ has been plotted for a system having almost an order of magnitude more monomers $(N=8000)$ for $C=80 \mu \mathrm{M}$ and $\epsilon=10$. Data points for both values of $N$ almost overlap on each other and therefore the conclusions made in our analyses seems to be unaffected by the finite-size effects.

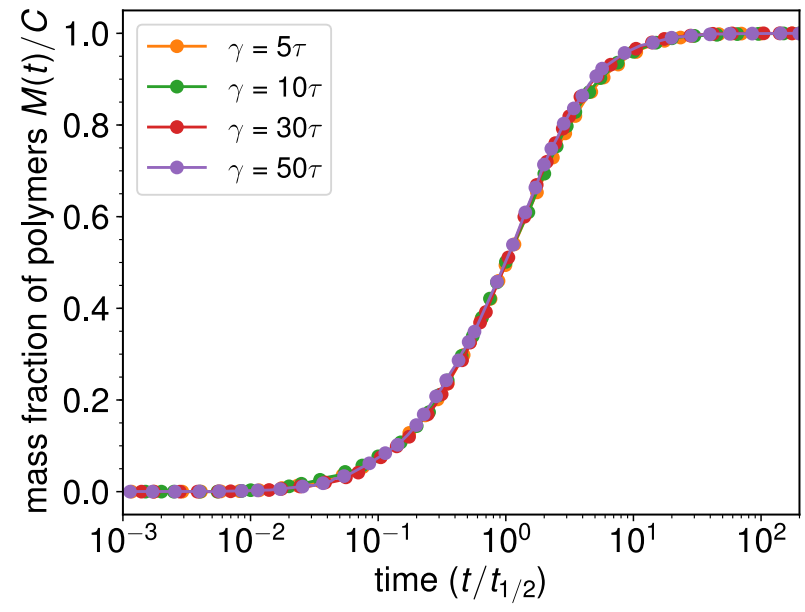

Supplemental Figure 8: Friction coefficient and polymerization. Polymerization curves for varying values of the friction coefficient $\gamma$. Results are for $C=40 \mu \mathrm{M}$ and $\epsilon=10$. 


\section{Effect of damping constant on the dynamics}

The MD simulations have been performed with LAMMPS ${ }^{2}$ using the Langevin thermostat representing the implicit solvent. In our simulations, the friction coefficient is chosen to be $\gamma=5 \tau$, basically setting the time scale for single monomer diffusion. In Supplemental Figure 8 , we confirm that changing the friction coefficient over one magnitude only rescales this time so that polymerization profiles collapse when plotted as a function of reduced time $t / t_{1 / 2}$.

\section{Helical transition}

\section{Description of shape and transition}

To describe the polymer shape, we borrow a representation developed for the modeling of DNA. ${ }^{3,4}$ To this end, we employ a local orthonormal frame with unit vectors $\boldsymbol{e}_{i}(k)$ for monomer $k$ such that $\boldsymbol{e}_{3}$ is the normal vector of the plane spanned by the three arms, and $\boldsymbol{e}_{1}$ points along one arm (cf. Supplemental Figure 9(a)). The shape of the polymer can then be described through rotation vectors $\boldsymbol{\omega}(k)=\sum_{i=1}^{3} \omega_{i}(k) \boldsymbol{e}_{i}(k)$ connecting adjacent frames

$$
\boldsymbol{e}_{i}(k+1) \approx \boldsymbol{e}_{i}(k)+\boldsymbol{\omega}(k) \times \boldsymbol{e}_{i}(k)
$$

(a)

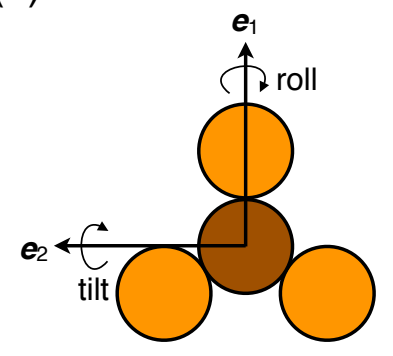

(b)

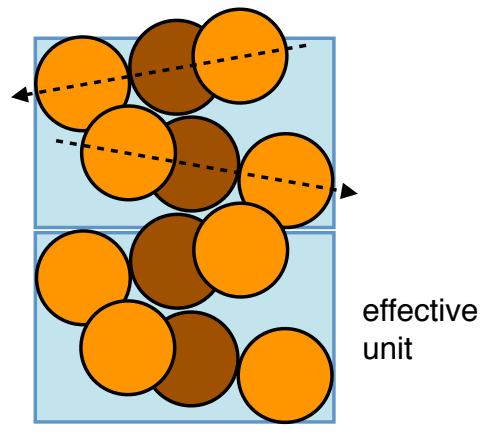

Supplemental Figure 9: (a) Top view showing central bead (dark) and inner beads (light) without terminal beads. Indicated is the local orthonormal frame with $\boldsymbol{e}_{3}$ pointing out of the plane. (b) Side view sketching the tilting between consecutive monomers. The arrows indicate $\boldsymbol{e}_{1}$. 
(a)
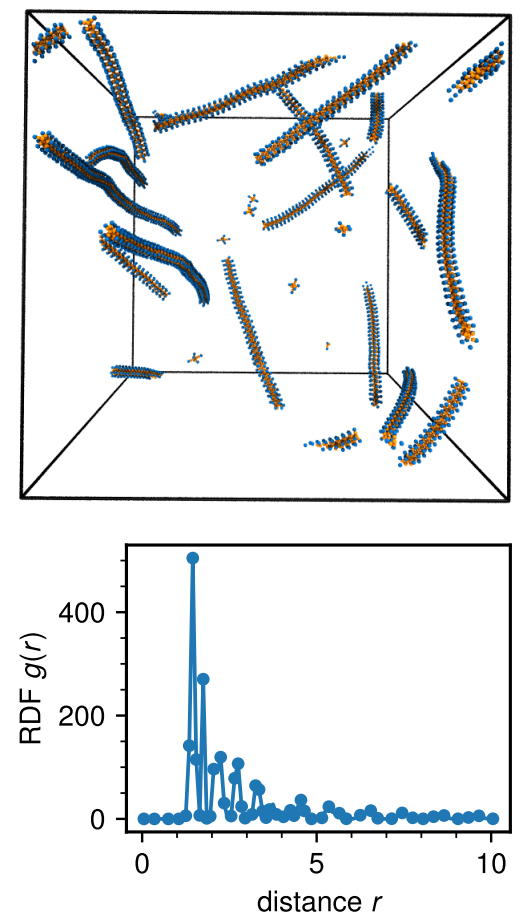

(b)
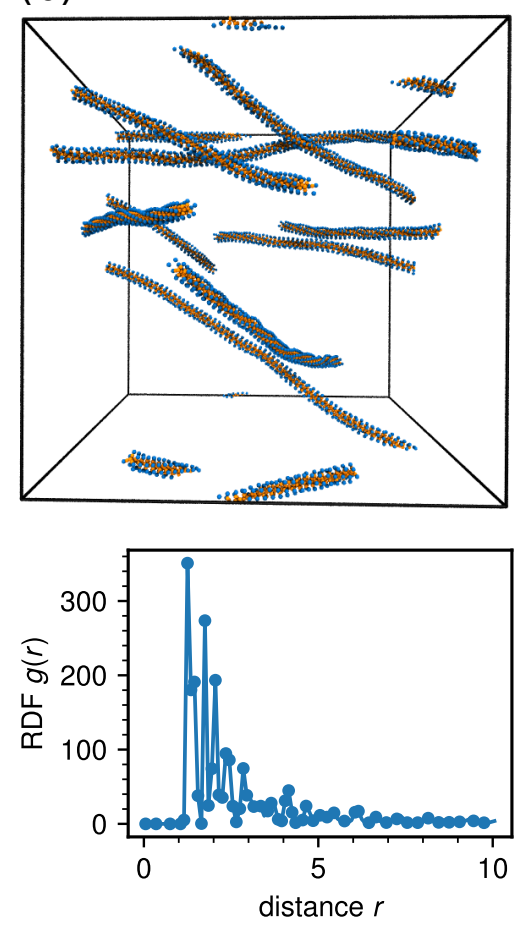

(c)
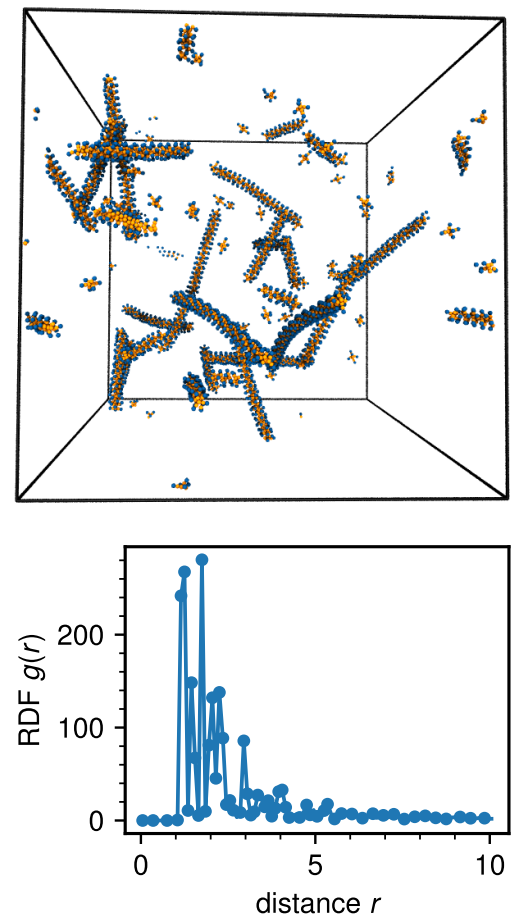

Supplemental Figure 10: Upper row: Final simulation snapshots for (a) $\epsilon=10<\epsilon_{c}$, (b) $\epsilon=$ $26 \simeq \epsilon_{c}$, and (c) $\epsilon=30>\epsilon_{c}$ across the transition. In (c), both left-handed and right-handed filaments are present. Bottom row: Corresponding radial distribution functions $g(r)$ of inner beads.

assuming small angles $\omega_{i}$. Here, the angles $\omega_{1}$ and $\omega_{2}$ correspond to roll and tilt, respectively, and $\omega_{3}=\phi$ corresponds to twisting.

In Supplemental Figure 10, we show snapshots taken from the end of simulation runs for three values of $\epsilon$ across the transition. Also shown are radial distribution functions of inner beads (excluding terminal and the central beads, cf. Supplemental Figure 9(a)). Notable is a shift of the first peak to smaller distances for helical polymers, i.e., these beads have moved closer on average. Combined with the observation that the separation of central beads remains approx. unchanged, we conclude that consecutive planes of monomers become tilted as sketched in Supplemental Figure 9(b).

Supplemental Figure 11(a) shows a single supramolecular polymer from the simulations at $\epsilon=28$. For clarity, now only every second monomer is shown. Alternating monomers have tilt, roll, and twist (rotations around all local orthogonal coordinates). Moreover, the 
(a)

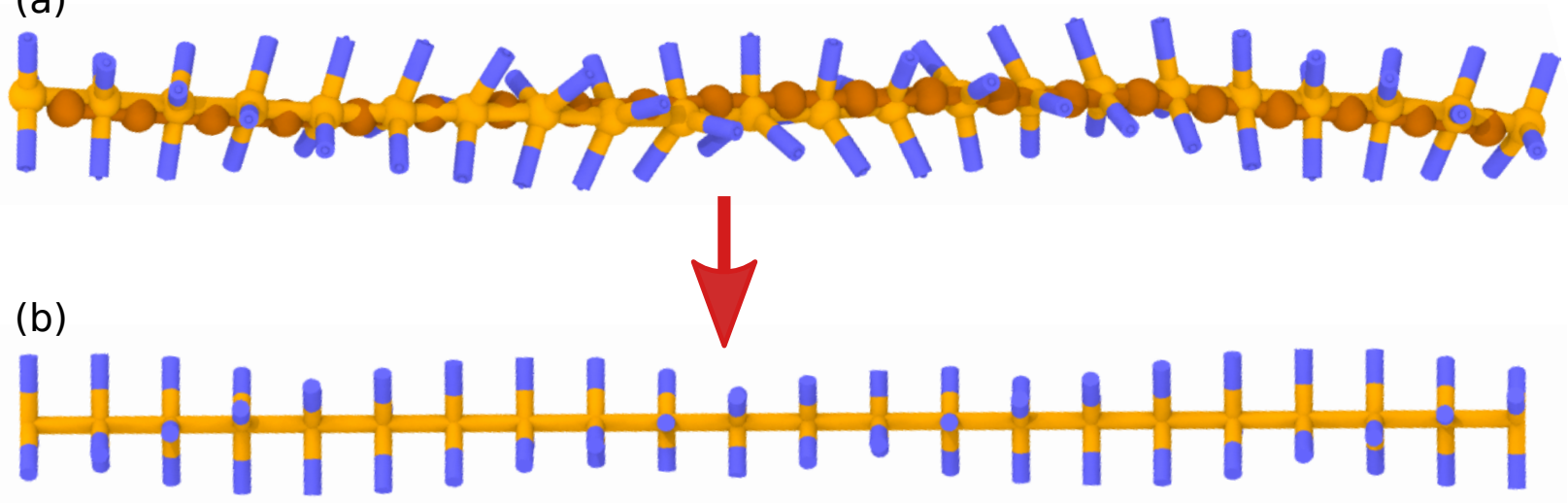

Supplemental Figure 11: (a) Snapshot of a helical filament showing only alternating monomers. We observe a non-zero tilt, roll, and twist (rotations around all local orthogonal coordinates). Moreover, the backbone of the helix (comprising the central beads of the alternating monomers) forms a double helix with the other series of alternating monomers.

(b) Tilt and roll have been removed to estimate the angle of twist between alternating monomers.

backbone of the helix, comprising the the central beads, forms a double helix with the other series of alternating monomers.

As order parameter, we are interested in the average angle of twist $\langle\phi\rangle$. To extract this angle from simulations, we remove roll and tilt as sketched in Supplemental Figure 11(b). We then calculate the difference $\phi$ of arm orientations between all consecutive alternating monomers and all polymers in the system, from which we estimate the average $\langle\phi\rangle$.

\section{Spontaneous symmetry breaking}

To understand the mechanism through which a helical superstructure with non-zero $\langle\phi\rangle$ can emerge, we combine two consecutive monomers (with alternate arm orientations) into one unit (sketched in Supplemental Figure 9(b)). We again employ local orthonormal frames but now for the units. We assume that the energy minimum is given by vanishing deformations with $\omega_{1}=\omega_{2}=\phi=0$. Expanding the energy around this straight configuration leads to

$$
\mathcal{E}=\sum_{k=1}^{N / 2} E(\boldsymbol{\omega}(k)), \quad E(\boldsymbol{\omega})=\frac{1}{2}\left[A\left(\omega_{1}^{2}+\omega_{2}^{2}\right)+C_{2} \phi^{2}+C_{4} \phi^{4}+2 G \omega_{2} \phi\right]
$$


with non-negative coefficients $A, C_{2}$, and $C_{4}$. Since $\boldsymbol{e}_{1}$ is a symmetry axis, the energy has to be invariant with respect to $\omega_{1} \rightarrow-\omega_{1} \cdot{ }^{3}$ However, to lowest order a term $\propto \omega_{2} \phi$ is possible, describing the coupling of tilt and twist between consecutive units, with coefficient $G$.

We now include the effect of thermal fluctuations. Due to the additivity of the total energy Eq. (2) it is sufficient to consider a single transformation between consecutive units. The partition function reads

$$
Z(\phi)=\int d \omega_{1} \int d \omega_{2} e^{-E} \sim \exp \left\{-\frac{1}{2}\left(C_{2}-\frac{G^{2}}{A}\right) \phi^{2}-C_{4} \phi^{4}\right\}
$$

where we have extended the integration limits to infinity and performed the Gaussian integrals. The minima of the resulting free energy

$$
F(\phi)=-\ln Z(\phi)=\frac{1}{2}\left(C_{2}-\frac{G^{2}}{A}\right) \phi^{2}+C_{4} \phi^{4}
$$

yield the average twist angle

$$
\langle\phi\rangle= \begin{cases}0 & |G| \leqslant G_{*} \\ \pm \sqrt{\frac{G^{2}-G_{*}^{2}}{4 C_{4} A}} & |G|>G_{*}\end{cases}
$$

with critical twist-tilt coupling $G_{*}=\sqrt{C_{2} A}$. The coefficients $A$ and $C_{2}$ are dominated by the steric interactions and thus independent of $\epsilon$, whereas $G$ should depend on $\epsilon$. We thus qualitatively reproduce the behavior shown in Figure 4 in the main text. Our derivation highlights that the emergence of a helical superstructure is an entropic effect: shape fluctuations become restricted when increasing the coupling $G$ until it is beneficial to adopt a non-zero $\langle\phi\rangle$. 


\section{Towards rational designing of monomers}

(a)

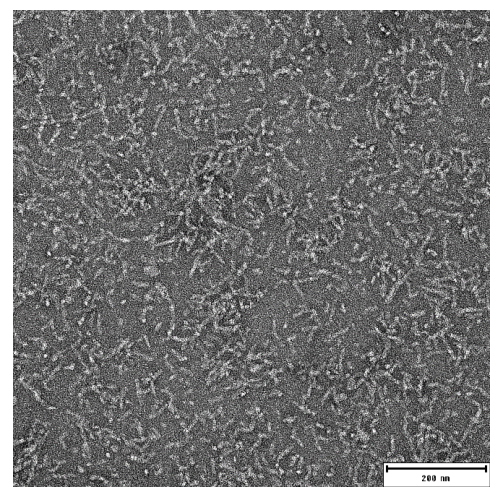

(d)

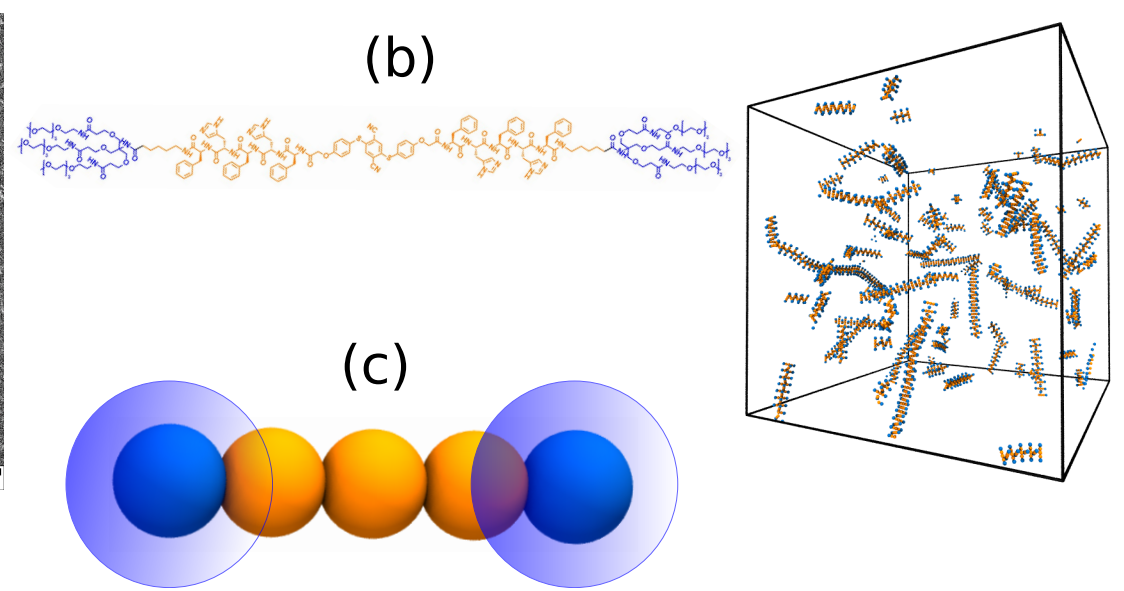

Supplemental Figure 12: Self-assembly of monomers with lower symmetry. (a) Negative stained TEM image of filaments. ${ }^{5}$ Solutions were prepared using $50 \mu \mathrm{M}$ monomer concentration in $10 \mathrm{mM}$ TRIS buffer $\left(\mathrm{pH} \mathrm{7.4)}\right.$ at $20^{\circ} \mathrm{C}$. Scale bar: $200 \mathrm{~nm}$. (b) chemical structure of the 2,5-bis-(phenylthio)phthalonitrile functionalized with two FHFHF pentapeptide sequences $(\mathrm{F}=$ phenyl alanine, $\mathrm{H}=$ histidine) and terminal dendritic tetraethylene glycol chains. ${ }^{5}$ (c) Schematic of the model in simulations. Blue shaded regions represent effective volume exclusion of the terminal beads. (d) Simulation snapshot shows filament formation of $C_{2}$-symmetric monomers, designed based on the principles of successful assembly of $C_{3}$-symmetric monomers. Here, $\epsilon=10$ and $C=40 \mu \mathrm{M}$. See text for the details of the model.

Non-directional, short range, attractive interaction along with steric hindrance is able to generate filaments of $C_{3}$-symmetric monomers from its dilute solutions. These principles are not restricted to this specific symmetry. Figure 12 shows formation of filaments of $C_{2}$-symmetric (rod-like) monomers. ${ }^{5}$ The monomer is comprised of five beads in a rigid linear geometry. Similar to the $C_{3}$-symmetric case, inner beads (orange) interact via short-range, isotropic, attractive interaction represented by Lennard-Jones potential (Eq. (2) in main article) and terminal beads (blue) only have volume exclusion (represented by WCA potential). Some modifications have been made in the parameters of the interaction potentials. Inner core beads have potential minima at $2^{1 / 6}(1.2 \sigma)$ with cut-off radius $4 \sigma$. The excluded volume has linear dimension $2.5 \sigma$. 


\section{References}

1. Oosawa, F.; sakura, S. Thermodynamics of the polymerization of protein; Academic Press, 1975.

2. Plimpton, S. Fast Parallel Algorithms for Short-Range Molecular Dynamics. Journal of Computational Physics 1995, 117, 1-19.

3. Marko, J. F.; Siggia, E. D. Bending and twisting elasticity of DNA. Macromolecules 1994, 27, 981-988.

4. Nomidis, S. K.; Skoruppa, E.; Carlon, E.; Marko, J. F. Twist-bend coupling and the statistical mechanics of the twistable wormlike-chain model of DNA: Perturbation theory and beyond. Phys. Rev. E 2019, 99, 032414.

5. Ahlers, P.; Götz, C.; Riebe, S.; Zirbes, M.; Jochem, M.; Spitzer, D.; Voskuhl, J.; Basché, T.; Besenius, P. Structure and luminescence properties of supramolecular polymers of amphiphilic aromatic thioether-peptide conjugates in water. Polym. Chem. 2019, 10, 3163-3169. 